are $\infty^{1}$ such inscribed and circumscribed pyramids $P$ the mapping upon $(x)$ gives the following theorem.

Theorem. Let $C_{4}^{\prime}$ and $C_{4}^{\prime \prime}$ be two fixed symmetric quartics in $(x)$. Construct a quartic $C_{4}{ }^{(1)}$ with a sextuple contact with $C_{4}^{\prime \prime}$, cutting $C_{4}^{\prime}$ in two sextuples $S_{1}$ and $S_{2}$. Through $S_{2}$ draw another quartic $C_{4}{ }^{(2)}$ with a sextuple contact with $C_{4}^{\prime \prime}$, which cuts $C_{4}^{\prime}$ in another sextuple $S_{3}$. Through $S_{3}$ draw similarly a third quartic $C_{4}{ }^{(3}$, cutting $C_{4}^{\prime}$ in a sextuple $S_{4}$, and so forth. Suppose that after drawing $n$ such quartics, the last $C_{4}{ }^{(n)}$ through $S_{n}$ cuts $C_{4}^{\prime}$ in a sextuple $S_{n+1}$ which coincides with $S_{1}$. If this happens once then there exists an infinite number of such series of quartics with the closure property.

Moreover there exist two other fixed quartics $D_{4}^{\prime}$ and $D_{4}^{\prime \prime}$ which are related to these series in precisely the same manner as $C_{4}^{\prime}$ and $C_{4}{ }^{\prime}$.

The UnIVERsity of ILLINoIS

\title{
CONGRUENCES OF LINES OF SPECIAL ORIENTATION RELATIVE TO A SURFACE OF REFERENCE*
}

BY M. C. FOSTER

1. Introduction. With each line $l$ of a rectilinear congruence let us associate the point $M$ in which $l$ intersects a surface of reference $S$. We refer $S$ to any orthogonal system. Let $\alpha, \beta, \gamma$ be the direction-cosines of $l$ relative to the moving trihedral of $S$ at $M$, the $x$-axis being chosen tangent to the curve $v=$ const. By congruences of special orientation relative to $S$, we shall mean those congruences for which the functions $\alpha, \beta, \gamma$ are of a special form. The present paper is concerned primarily with the case when $\alpha, \beta, \gamma$ are constant.

2. Normal Congruences. Relative to the moving trihedral the coordinates of any point $P$ on $l$ are

\footnotetext{
* Presented to the Society, December 28, 1926.
} 


$$
x=\alpha t, \quad y=\beta t, \quad z=\gamma t,
$$

where $t$ is the distance along $l$ from $M$ to $P$. The condition that there exist a surface $\Sigma$ normal to $l$ is that the displacements of some point on $l$ satisfy the relation

$$
\sum \alpha \delta x=0,
$$

for all values of $d v / d u$. This becomes on using the well known formulas*

$$
\frac{\partial t}{\partial u} d u+\frac{\partial t}{\partial v} d v+\alpha \xi d u+\beta \eta_{1} d v \equiv 0
$$

Hence

$$
\frac{\partial t}{\partial u}+\alpha \xi=0, \quad \frac{\partial t}{\partial v}+\beta \eta_{1}=0 .
$$

The condition of integrability,

$$
\frac{\partial}{\partial v}(\alpha \xi)=\frac{\partial}{\partial u}\left(\beta \eta_{1}\right)
$$

is therefore a necessary condition that the congruence be normal. It is also sufficient. For if (5) is satisfied, the function $t$ as given by (4) will satisfy (3), and consequently there exists a one-parameter family of surfaces $\Sigma$ normal to the congruence.

Let us now assume that

$$
\alpha=U, \quad \beta=V,
$$

where $U$ and $V$ are functions of $u$ and $v$ alone respectively. The relation (5) becomes

$$
U \frac{\partial \xi}{\partial v}=V \frac{\partial \eta_{1}}{\partial u}
$$

which may be written

$$
U \eta_{1} r=-V \xi r_{1}
$$

* Eisenhart, Differential Geometry of Curves and Surfaces, p. 170.

$\dagger$ Eisenhart, p. 170. 
or

$$
-\frac{U}{V}=\frac{\xi r_{1}}{\eta_{1} r}=\frac{\rho_{g u}}{\rho_{g v}},
$$

where $\rho_{g u}$ and $\rho_{g v}$ are the radii of geodesic curvature of the curves $v=$ const. and $u=$ const., respectively. From (8) we have the following theorem.

Theorem 1. If the lines $l$ be of fixed orientation relative to the trihedral of $S$, a necessary and sufficient condition that the congruence be normal is that the corresponding radii of geodesic curvature of the curves $v=$ const. and $u=$ const., respectively, be in the constant ratio $\alpha:-\beta$.

The condition that the curves $v=$ const., and $u=$ const., be geodesics is that $r=0$, and $r_{1}=0$, respectively. Hence from (7) we have the following theorems.*

THEOREM 2. If $\alpha,(\beta)$, be zero, a necessary and sufficient condition that the congruence be normal is that the curves $u=$ const., $(v=$ const. $)$, be geodesics.

THEOREM 3. If the curves $u=$ const., ( $v=$ const.), be geodesics, a necessary and sufficient condition that the congruence be normal is that either the curves $v=$ const., $(u=$ const. $)$, be geodesics and $S$ be developable, or $\alpha,(\beta)$, be zero.

We note that Theorem 2 includes as a special case the well known theorem that a necessary and sufficient condition that the tangents to a family of curves on a surface constitute a normal congruence is that these curves be geodesics.

3. Equation Defining the Developables. We shall assume for the moment that $\alpha, \beta, \gamma$ are any functions whatever of $u$ and $v$. For $l$ to generate a developable surface the displacement of some point on $l$ must satisfy the relations

or

$$
\frac{\delta x}{\alpha}=\frac{\delta y}{\beta}=\frac{\delta z}{\gamma},
$$

$$
\alpha \delta z-\gamma \delta x=0, \quad \beta \delta z-\gamma \delta y=0 .
$$

* We exclude the case where the lines are normal to $S$. 
When $t$ is eliminated between these equations we get the following equation for the curves on $S$ defining the developables,

$$
\left|\begin{array}{ccc}
\alpha & \xi d u & A_{1} d u+A_{2} d v \\
\beta & \eta_{1} d v & B_{1} d u+B_{2} d v \\
\gamma & 0 & C_{1} d u+C_{2} d v
\end{array}\right|=0
$$

where we have set

$$
\begin{cases}A_{1}=\frac{\partial \alpha}{\partial u}+q \gamma-r \beta, & A_{2}=\frac{\partial \alpha}{\partial v}+q_{1} \gamma-r_{1} \beta \\ B_{1}=\frac{\partial \beta}{\partial u}+r \alpha-p \gamma, & B_{2}=\frac{\partial \beta}{\partial v}+r_{1} \alpha-p_{1} \gamma \\ C_{1}=\frac{\partial \gamma}{\partial u}+p \beta-q \alpha, & C_{2}=\frac{\partial \gamma}{\partial v}+p_{1} \beta-q_{1} \alpha\end{cases}
$$

When $d v / d u$ is eliminated between equations (9) we get the following equation in $t$ for the distances to the focal points:

$$
\left|\begin{array}{ccc}
\alpha & A_{1} t+\xi & A_{2} t \\
\beta & B_{1} t & B_{2} t+\eta_{1} \\
\gamma & C_{1} t & C_{2} t
\end{array}\right|=0 .
$$

4. Distance to a Line of Striction. The direction-cosines

$$
\alpha+\delta \alpha, \quad \beta+\delta \beta, \quad \gamma+\delta \gamma
$$

of a neighboring line $l^{\prime}$ of the congruence are found by considering the displacements $\delta \alpha, \delta \beta, \delta \gamma$ of a point $(\alpha, \beta, \gamma)$ on the unit sphere, relative to a trihedral of fixed vertex at the center of the unit sphere, whose axes are parallel to the axes of the moving trihedral. If a point $P$ on $l$ generate the line of striction of the ruled surface defined by a value of $d v / d u$, the displacement of $P$ must be orthogonal to both $l$ and $l^{\prime}$. Hence

$$
\sum \alpha \delta x=0, \quad \sum(\alpha+\delta \alpha) \delta x=0
$$


or

$$
\sum \delta \alpha \delta x=0 .
$$

This becomes, by means of well known formulas,

$$
\left\{\begin{aligned}
& \left\{\frac{\partial \alpha}{\partial u} d u+\frac{\partial \alpha}{\partial v} d v+\gamma\left(q d u+q_{1} d v\right)-\beta\left(r d u+r_{1} d v\right)\right\} \\
\cdot & \left\{\left(\frac{\partial \alpha}{\partial u} d u+\frac{\partial \alpha}{\partial v} d v\right) t+\xi d u+\gamma t\left(q d u+q_{1} d v\right)\right. \\
& \left.-\beta t\left(r d u+r_{1} d v\right)\right\} \\
+ & \left\{\frac{\partial \beta}{\partial u} d u+\frac{\partial \beta}{\partial v} d v+\alpha\left(r d u+r_{1} d v\right)-\gamma\left(p d u+p_{1} d v\right)\right\} \\
\cdot & \left\{\left(\frac{\partial \beta}{\partial u} d u+\frac{\partial \beta}{\partial v} d v\right) t+\eta_{1} d v+\alpha t\left(r d u+r_{1} d v\right)\right. \\
& \left.-\gamma t\left(p d u+p_{1} d v\right)\right\} \\
+ & \left\{\frac{\partial \gamma}{\partial u} d u+\frac{\partial \gamma}{\partial v} d v+\beta\left(p d u+p_{1} d v\right)-\alpha\left(q d u+q_{1} d v\right)\right\} \\
\cdot & \left\{\left(\frac{\partial \gamma}{\partial u} d u+\frac{\partial \gamma}{\partial v}\right) t+\beta t\left(p d u+p_{1} d v\right)\right. \\
& \left.-\alpha t\left(q d u+q_{1} d v\right)\right\}=0 .
\end{aligned}\right.
$$

If a value be assigned to $t$ this equation gives us the equation of the curves on $S$ defining the ruled surfaces for which $t$ is the distance to their lines of striction; and if a value be assigned to $d v / d u$ we have an equation in $t$ which gives us the distance to the line of striction of the ruled surface so determined.

5. The Direction Cosines $\alpha, \beta, \gamma$, constant. We now assume the lines of the congruence to be of fixed orientation relative to the moving trihedral. The curves defining the ruled surfaces whose lines of striction lie on $S$ will be found by setting $t=0$ in (13). We get, for the equation of these curves, 


$$
\begin{gathered}
\xi(\beta r-\gamma q) d u^{2}+\left\{\xi\left(\beta r_{1}-\gamma q_{1}\right)+\eta_{1}(\gamma p-\alpha r)\right\} d u d v \\
+\eta_{1}\left(\gamma p_{1}-\alpha r_{1}\right) d v^{2}=0
\end{gathered}
$$

The curves $v=$ const., or $u=$ const., will be the lines of striction of the ruled surfaces which they define if

$$
\beta r-\gamma q=0
$$

or

$$
\gamma p_{1}-\alpha r_{1}=0
$$

respectively. If

$$
\beta r-\gamma q=0
$$

we have*

$$
\frac{\beta}{\gamma}=\text { const. }=\frac{q}{r}=-\operatorname{ctn} \omega_{u},
$$

where $\omega_{u}$ is the angle between the positive principal normal of the curve $v=$ const., and the positive normal to $S$, the angle being measured toward the positive binormal. Hence we have the following theorem.

THEOREM 4. If $l$ be of fixed orientation relative to the trihedral, a necessary and sufficient condition that the curves $v=$ const. be the lines of striction of the ruled surfaces which they define is that their osculating planes meet the normal under the constant angle defined by (15).

Let us here recall a theorem of ruled surfaces due to Bonnet:

If a curve upon a ruled surface have two of the following properties, it has the third also:

1. that it cut the rulings under constant angle,

2. that it be the line of striction,

3. that it be a geodesic.

Hence Theorem 4 may be extended to state that the curves $v=$ const. are geodesics on the ruled surfaces which they

\footnotetext{
* Eisenhart, p. 167.
} 
define; and the principal normals meet the rulings orthogonally. A similar theorem holds for the curves $u=$ const.

The condition that $S$ be the middle surface is that the coefficient of $t$ in (12) shall vanish. We get as this condition

$$
\begin{array}{r}
\xi p_{1}\left(\alpha^{2}-1\right)+\eta_{1} q\left(1-\beta^{2}\right)+\alpha \beta\left(\xi q_{1}-\eta_{1} p\right) \\
+\gamma\left(\alpha \xi r_{1}-\beta \eta_{1} r\right)=0 .
\end{array}
$$

If $S$ be minimal we have $\xi p_{1}-\eta_{1} q=0$; consequently, if $\alpha=\beta$, and $S$ be referred to its lines of curvature, (16) reduces to

or

$$
\xi r_{1}=\eta_{1} r
$$

$$
\rho_{g u}=\rho_{g v} .
$$

Hence we have the following theorem.

THEOREM 5. If $S$ be a minimal surface referred to its lines of curvature, a necessary and sufficient condition that the congruence of fixed orientation for which $\alpha=\beta$, have $S$ as its middle surface, is that the corresponding radii of geodesic curvature of the parametric curves be equal.

We note from (7) that such congruences are not normal.

Let the lines of curvature be parametric. The condition that the developables of the congruence be represented on $S$ by the parametric system is that the coefficients of $d u^{2}$ and $d v^{2}$ in (9) shall vanish. This condition is

$$
\alpha C_{2}-\gamma A_{2}=0, \quad \gamma B_{1}-\beta C_{1}=0 .
$$

Hence

$$
\left\{\begin{aligned}
-\frac{\alpha}{\gamma} & =\text { const. }=\frac{r_{1}}{p_{1}}=\tan \omega_{v}, \\
\frac{\beta}{\gamma} & =\text { const. }=-\frac{r}{q}=\tan \omega_{u} .
\end{aligned}\right.
$$

Consequently, the osculating planes of the lines of curvature meet the tangent planes under constant angle, and they are 
therefore plane curves. ${ }^{*}$ We have in consequence the following theorem.

THEOREM 6. If $S$ be referred to its lines of curvature, a necessary and sufficient condition that the developables of the congruence of lines of fixed orientation be defined by the parametric curves is that these curves be plane in both systems, and that their osculating planes meet the normals under the constant angles defined by (18).

When relations (18) are substituted in (16), this condition that $S$ be the middle surface reduces to

$$
\xi p_{1}-\eta_{1} q=0 .
$$

Hence if we further assume $S$ to be the middle surface, it must also be minimal. We note that in this case the congruence is of the Ribaucour type; for the developables intersect the middle surface in a conjugate system.

6. Isotropic Congruences. We shall again assume for the moment that $\alpha, \beta, \gamma$ are any functions whatever of $u$ and $v$. The necessary and sufficient condition that a congruence be isotropic is that all the lines of striction lie on the middle surface. Evidently if all lines of striction lie on some surface, that surface is the middle surface. We suppose that all lines of striction lie on $S$. Then from (13) we must have

$$
t \equiv 0,
$$

or

$$
A_{1}=0, \quad \xi A_{2}+\eta_{1} B_{1}=0, \quad B_{2}=0 .
$$

Hence when $S$ is the middle surface (19) represents the necessary and sufficient condition that a congruence be isotropic.

We again assume $\alpha, \beta, \gamma$ constant. The relations (19) become

$$
\left\{\begin{array}{l}
q \gamma-r \beta=0 \\
\xi\left(q_{1} \gamma-r_{1} \beta\right)+\eta_{1}(r \alpha-p \gamma)=0 \\
r_{1} \alpha-p_{1} \gamma=0
\end{array}\right.
$$

\footnotetext{
* Eisenhart, p. 150.
} 
from which we have

$$
\left\{\begin{array}{l}
\frac{\gamma}{\beta}=\text { const. }=\frac{r}{q}=-\tan \omega_{u}, \\
\frac{\gamma}{\alpha}=\text { const. }=\frac{r_{1}}{p_{1}}=\tan \omega_{v} .
\end{array}\right.
$$

Using the first and third equations of (20) the second member reduces to

$$
\eta_{1} p_{1} r^{2}-\xi q r_{1}^{2}+2 \xi q_{1} r r_{1}=0 .
$$

From (21) we see that the osculating planes of the parametric curves meet the normals under constant angles. We have therefore the following theorem.

THEOREM 7. A condition that the congruence of lines of fixed orientation relative to its middle surface be an isotropic congruence, is that the osculating planes of the parametric curves meet the normals under the constant angles defined by (21), and that the fundamental quantities of the middle surface satisfy (22).

7. Congruences of Ribaucour. The condition that a congruence be of the Ribaucour type is that its developables meet the middle surface in a conjugate system. We assume $S$ to be the middle surface referred to its lines of curvature. From (10) we readily find the condition that the curves defining the developables form a conjugate system; this reduces to

$$
p_{1} r \alpha-q r_{1} \beta=0 .
$$

Hence when $S$ is taken as the middle surface referred to its lines of curvature, the relation (23) represents the necessary and sufficient condition that a congruence of fixed orientation relative to $S$ be of the Ribaucour type. This condition is readily reducible to

$$
\frac{\alpha}{\beta}=\text { const. }=-\tan \bar{\omega}_{v} \operatorname{ctn} \bar{\omega}_{\mu} .
$$


8. Condition that the Lines of Curvature on $S$ and on the Normal Surfaces be in Correspondence. We have seen in $\$ 5$ that when the lines of curvature on $S$ are parametric, a condition that the developables be represented by the parametric curves is

$$
\frac{\alpha}{\gamma}=-\frac{r_{1}}{p_{1}}, \quad \frac{\gamma}{\beta}=-\frac{q}{r} .
$$

From these we get

$$
\frac{\alpha}{\beta}=\frac{r_{1} q}{p_{1} r} .
$$

When the congruence is normal we have, from (7),

$$
\frac{\alpha}{\beta}=-\frac{\xi r_{1}}{\eta_{1} r} ;
$$

and from (24) and (25) we have

$$
\xi p_{1}+\eta_{1} q=0 .
$$

Hence, when $S$ is referred to its lines of curvature, the relation (26) represents a condition that the parametric curves on $S$ correspond to the lines of curvature on the surfaces normal to the congruence of fixed orientation.

Wirliams College 